\title{
Single-drop impingement onto a wavy liquid film and description of the asymmetrical cavity dynamics
}

\author{
Nils Paul van Hinsberg ${ }^{1, *}$ and Marie Charbonneau-Grandmaison ${ }^{2, \dagger}$ \\ ${ }^{1}$ Institute of Aeroelasticity, German Aerospace Center, Bunsenstraße 10, 37073 Göttingen, Germany \\ ${ }^{2}$ Institute of Fluid Mechanics and Aerodynamics, Technische Universität Darmstadt, Petersenstraße 30, 64287 Darmstadt, Germany
}

(Received 8 January 2015; revised manuscript received 26 March 2015; published 6 July 2015)

\begin{abstract}
The present paper is devoted to an experimental investigation of the cavity formed upon a single-drop impingement onto a traveling solitary surface wave on a deep pool of the same liquid. The dynamics of the cavity throughout its complete expansion and receding phase are analyzed using high-speed shadowgraphy and compared to the outcomes of drop impingements onto steady liquid surface films having equal thickness. The effects of the surface wave velocity, amplitude and phase, drop impingement velocity, and liquid viscosity on the cavity's diameter and depth evolution are accurately characterized at various time instants. The wave velocity induces a distinct and in time increasing inclination of the cavity in the wave propagation direction. In particular for strong waves an asymmetrical distribution of the radial expansion and retraction velocity along the cavity's circumference is observed. A linear dependency between the absolute Weber number and the typical length and time scales associated with the cavity's maximum depth and maximum diameter is reported.
\end{abstract}

DOI: 10.1103/PhysRevE.92.013004 PACS number(s): 47.15.gm, 47.35.Fg, 47.55.D-, 47.55.nd

\section{INTRODUCTION}

The fluid mechanics behind impingements of drops on dry or (partially) wetted surfaces has a great relevance in a large variety of technical, ecological, agricultural, and meteorological fields. Specific applications can for example be found inside gas turbines, for spray drying, coating of metallic products [1] and cooling, in medical applications and forensic investigations, as well as the electrification inside waterfalls [2], during thunderstorms [3], soil erosion [4], and in the dispersion of seed and microorganisms. For most of these applications the splashing of drops on thin liquid layers, the latter ones possessing a morphology that is both locally and globally highly dynamic, influences the flow behavior.

The impingement of isolated drops onto various steady surfaces has been thoroughly investigated in many aspects both experimentally, numerically, and theoretically (see, for example, [5] for a broad overview). The influences of the drop oscillation before impingement were for example investigated in [6] and [7], whereas the effects of these oscillations on the vortex rings formed at the tip of the crater were described in [8-10]. The latter phenomenon was also studied in [11] by dying the impacting drop for a clear distinction between impinging drop and film liquid. By using high-speed visualization they found that the vortex ring structure actually consists of two substructures: an outer and a central vortex ring. They furthermore used the laser Doppler velocimetry

\footnotetext{
*nils.vanhinsberg@dlr.de

${ }^{\dagger}$ Present address: Institute of Robotics and Intelligent Systems, LEO D 9.2, Swiss Federal Institute of Technology Zürich, 8092 Zürich ETH-Zentrum, Switzerland.
}

Published by the American Physical Society under the terms of the Creative Commons Attribution 3.0 License. Further distribution of this work must maintain attribution to the author(s) and the published article's title, journal citation, and DOI. technique to measure the velocity distributions at various points below the cavity, thereby presenting time-dependent axial and radial film velocities upon single-drop impingement. Several authors [12-15] investigated the concentric capillary waves that are formed at the gas-liquid interface after drop impingement. These waves travel in the downward direction along the sidewall of the cavity and change its shape from hemispherical to conical. In the case of deep pools the mergence of the capillary waves at the bottom of the cavity can lead to the entrapment of an air bubble as observed in [16-18] and the occurrence of underwater sound $[13,19,20]$. The effects of the impingement angle on the rebound and coalescence of single drops onto deep pools and on the total mass of secondary droplets were, among others, experimentally studied in [21-23]. Numerical simulations were performed by [24] to study the influences of the impingement angle, velocity, and target film height on the crater height and displacement, as well as on the crown height and angle after impingements onto a deep and shallow pool. In [20] and [25] it was proven that the cavity formation and collapse process is sensitive to a variation in the surface tension and viscosity of the used liquid. Based on this large amount of literature the single-drop impingement can nowadays be modeled relatively well [26-29].

To the best of authors' knowledge, however, no experimental or numerical data are available on the effects that the waviness and internal velocity components of the surface film may have on the dynamics of the cavity produced by an impinging drop. In view of this, the main subject of the present paper is the analysis of the experimental data of a drop impingement onto a traveling solitary surface wave. The evolution of the shape of the cavity in time, formed upon impingement, is investigated for various terminal drop impingement velocities, phases of the surface wave at impingement, wave amplifications (i.e., wave velocity and amplitude), and liquid properties. The study focuses on the time evolution of the depth and the diameter of the cavity during its penetration, expansion, receding, and retraction phase. 


\section{EXPERIMENTAL DESCRIPTION AND OPERATING SETTINGS}

\section{A. Experimental setup}

Experimental measurements were performed using the experimental setup shown in Fig. 1. Drops are generated using a medical syringe pump (a) that is programed to dispense the liquid at a constant flow rate in the range 0.40-1.81 $\mu \mathrm{l} / \mathrm{s}$, depending on the fluid used. The falling drops (b) are enclosed in a vertical cylinder of $32 \mathrm{~mm}$ in diameter to protect them from air flow disturbances. The drops pass a light barrier before impinging onto the liquid surface film (d), which is held inside a Perspex cube with a surface area of $100 \times 100 \mathrm{~mm}$ (c). In order to investigate the influence of the waviness of the surface film on the cavity dynamics, a Perspex plate (f) is positioned vertically inside the cube and connected to a vibration exciter (g) type 4809 (Brüel \& Kjær). This exciter allows a peak-to-peak vibration amplitude between 0 and $8 \mathrm{~mm}$ at a frequency range of $10-20 \mathrm{kHz}$. The exciter is controlled by a power amplifier, type 2718 (Brüel \& Kjær), as well as by a digital delay or pulse generator, type PDG-2510 (Directed Energy Inc.). With the amplifier the correct maximum amplitude of the horizontally moving plate can be set, whereas the delay generator adds a time delay to the signal coming from the photodiode and is used to set the plate into motion. In order to generate sinusoidal surface waves with different amplitudes and propagation velocities, a lever arm with a variable length is applied. In this way both the amplitude and the phase of the surface wave at the instant of the drop impingement can be set precisely, thereby assuring a clear repeatability of the studied impingement process. About 20 sec elapse between subsequent impingements to assure equal boundary conditions of the surface film before generating the next surface wave.

The light barrier also activates a second electronic delay circuit, that triggers the imaging system, consisting of a high-speed camera [Photron Fastcam SA1.1 model 675K-M1 (h)]. The camera images are recorded in a plane that is perpendicular to the travel direction of the surface wave, as the greatest impact of the surface wave on the cavity dynamics is to be expected in the wave direction. Its optical axis is aligned with the free surface of the quiet target liquid to avoid optical aberrations. An extended 85-mm Cosimar Television lens is used for a good balance between the required spatial resolution $-23.3 \mu \mathrm{m} /$ pix — and the necessary field of view

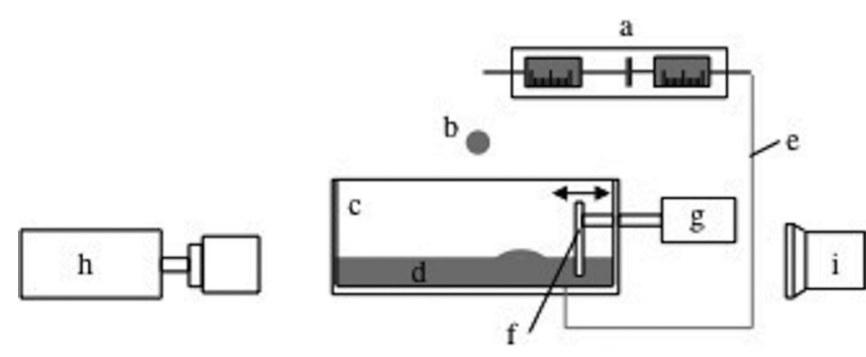

FIG. 1. Experimental setup. (a) Push-pull syringe pump, (b) impinging drop, (c) Perspex container, (d) surface film, (e) liquid suction channel, (f) translating plate, (g) exciter, (h) high-speed digital camera, (i) light source. of $569 \mathrm{~mm}^{2}$. All recordings are performed at a frequency of $5.4 \mathrm{kHz}$ and an exposure time of $1 \mu \mathrm{s}$. In order to calculate the drop velocity and diameter 20 recorded images just prior to the first contact between the drop and the liquid film are analyzed. By applying a MATLAB algorithm the position, the roundness and the center of the drop are located in each image, after which the mean and standard deviation of the drop diameter can easily be determined. Since the recording frequency of the camera is known, the mean drop velocity and standard deviation are calculated using the vertical displacement of the center of the drop between subsequent recordings. In a similar way the wave propagation velocity and wave amplitude are determined by tracking the wave front prior to impingement. Every recording sequence is repeated five times to verify the repeatability of the impingement outcomes.

\section{B. Physical properties and operating settings}

Comparison of different cases is enabled by the use of the corresponding nondimensional numbers governing the impact process: Weber number $\mathrm{We}=\rho U_{a b s}^{2} D / \sigma$, Reynolds number $\operatorname{Re}=U_{a b s} D / v$, Froude number $\operatorname{Fr}=U_{a b s}^{2} /(g D)$, the nondimensional film thickness $h^{*}=h / D$, the nondimensional wave amplitude $A_{w}^{*}=A_{w} / D$, and the nondimensional time $t^{*}=t U_{a b s} / D$. In the above expressions $\nu, \rho$, and $\sigma$ are the kinematic viscosity, density, and surface tension of the liquid, $D$ is the drop diameter, $h$ is the initial quiet surface film thickness, $A_{w}$ is the wave amplitude, and $t$ is the time, which is set to zero at the first contact between the impacting drop and the surface film. $U_{a b s}$ is the resultant overall impingement velocity of the drop and is defined as $U_{a b s}=\left(U_{d}^{2}+U_{w}^{2}\right)^{0.5}$. Hence, since the velocity of the wave $U_{w}$ is taken into account as well, the overall drop impingement velocity is slightly higher than the terminal impingement velocity component of the drop solely due to gravity, $U_{d}$.

In order to investigate the influence of the liquid properties on the cavity dynamics, two liquids are used: distilled water and a glycerine-water mixture consisting of $30 \%$ glycerine and $70 \%$ water. The physical properties of both liquids and the ranges of the aforementioned nondimensional numbers are given in Table I. Note that in this table all nondimensional numbers are based on the overall drop velocity $U_{a b s}$. The fluids of the impinging drops and of the target liquid are equal for all performed experiments. The diameters of the impinging drops are constant and have a value of $2.90 \pm 0.14$ for distilled water and $2.57 \pm 0.05$ for the glycerine-water mixture. Two kinds of surface waves having different amplitudes and velocities (see Table I) are generated inside the surface film to investigate the behavior of the cavity on the relative wave velocity and amplitude. The initial thickness of the quiet target film is kept constant by means of a push-pull syringe pump [(a) in Fig. 1], which sucks the same amount of liquid from the film [(e) in Fig. 1] as is added by the impacting drops, yielding constant nondimensional thicknesses of $\overline{h^{*}}=5.8$ and $\overline{h^{*}}=6.4$ with an error in the film thickness of less than $1 \%$ for both liquids. Further properties of the impinging drops and of the surface waves are listed in Table I. To obtain experimental data of the evolutions of the cavities after impingement onto different phases of the solitary waves, a variable time delay between the instant at which the impinging drop passes the photodiode and 
TABLE I. Physical properties of the liquids used $\left(\right.$ at $\left.30^{\circ} \mathrm{C}\right)$, the ranges of nondimensional numbers, and the wave properties for the single-drop impingement studies onto solitary surface waves.

\begin{tabular}{|c|c|c|}
\hline & Distilled water & Glycerine-water (30-70\% vol.) \\
\hline Density $\rho\left(\mathrm{kg} / \mathrm{m}^{3}\right)$ & 996 & 1069 \\
\hline Viscosity $\mu[\mathrm{kg} /(\mathrm{ms})]$ & $7.98 \times 10^{-4}$ & $1.86 \times 10^{-3}$ \\
\hline Surface tension $\sigma\left(\mathrm{kg} / \mathrm{s}^{2}\right)$ & $7.12 \times 10^{-2}$ & $6.68 \times 10^{-2}$ \\
\hline Ohnesorge number Oh & 0.0018 & 0.0019 \\
\hline Weber number We & $235-521$ & $134-413$ \\
\hline Reynolds number Re & $8300-13300$ & $7200-11000$ \\
\hline Froude number Fr & $210-410$ & $160-450$ \\
\hline Nondimensional undisturbed film thickness $\overline{h^{*}}$ & 5.8 & 6.4 \\
\hline Nondimensional wavy film thickness $h^{*}$ & $3.9-7.7$ & $4.3-8.5$ \\
\hline Wave velocity $U_{w}(\mathrm{~m} / \mathrm{s})$ & $0.311 \pm 0.003-0.392 \pm 0.003$ & $0.197 \pm 0.005-0.322 \pm 0.014$ \\
\hline Nondimensional wave velocity $U_{w}^{*}$ & $0.10-0.15$ & $0.07-0.13$ \\
\hline Wave amplitude $A_{w}(\mathrm{~mm})$ & $2.26 \pm 0.01-5.63 \pm 0.02$ & $1.77 \pm 0.01-5.49 \pm 0.01$ \\
\hline Nondimensional wave amplitude $A_{w}^{*}$ & $0.78-1.94$ & $0.69-2.14$ \\
\hline Drop diameter $D(\mathrm{~mm})$ & $2.90 \pm 0.14$ & $2.57 \pm 0.05$ \\
\hline Drop velocity ${ }^{\mathrm{a}} U_{d}(\mathrm{~m} / \mathrm{s})$ & $2.66 \pm 0.10-3.12 \pm 0.14$ & $2.50 \pm 0.29-2.99 \pm 0.19$ \\
\hline Overall drop velocity $U_{a b s}(\mathrm{~m} / \mathrm{s})$ & $2.68 \pm 0.10-3.14 \pm 0.14$ & $2.51 \pm 0.29-3.01 \pm 0.19$ \\
\hline
\end{tabular}

at impingement.

the generation of the solitary wave is set. These delay times are a function of the Weber number of the impinging drop and of the liquid properties and therefore have to be defined in advance. They are chosen in such a way that the impingement points are spread over the first half of the wavelength of the sinusoidal solitary wave, hence the upper half of the wave between $0^{\circ} \leqslant \varphi \leqslant 180^{\circ}$.

\section{RESULTS AND DISCUSSION}

This section focuses in detail on the results of the singledrop impingement experiments that are used to study the effect of traveling solitary surface waves on the diameter and depth evolution of the cavity in time. The results are presented both in a qualitative and in a quantitative manner. By comparing these results with those found for impingements onto steady

TABLE II. Parameters of the performed experiments used for the discussion of the impingement outcomes of the single-drop impingement upon solitary surface waves.

\begin{tabular}{|c|c|c|c|c|c|c|c|c|c|c|}
\hline Liquid & Amplified & $\overline{h^{*}}$ & We & Fr & $\operatorname{Re}$ & Phase $\varphi$ & $\bar{D}_{\text {cav }, \max }^{*}$ & $\bar{t}_{\text {diameter,max }}^{*}$ & $\overline{D e p t h}_{\max }^{*}$ & $\bar{t}_{\text {depth,max }}^{*}$ \\
\hline Distilled water & no & 5.8 & 304 & 251 & 10,411 & $26^{\circ} \leqslant \varphi \leqslant 146^{\circ}$ & $3.05^{\mathrm{a}}$ & $11.8^{\mathrm{a}}$ & $2.07^{\mathrm{a}}$ & $12.7^{\mathrm{a}}$ \\
\hline Distilled water & no & 5.8 & 378 & 269 & 11,611 & $21^{\circ} \leqslant \varphi \leqslant 134^{\circ}$ & $3.34^{\mathrm{a}}$ & $14.4^{\mathrm{a}}$ & $2.17^{\mathrm{a}}$ & $14.6^{\mathrm{a}}$ \\
\hline Distilled water & no & 5.8 & 398 & 328 & 11,458 & $31^{\circ} \leqslant \varphi \leqslant 162^{\circ}$ & $3.05^{\mathrm{a}}$ & $16.3^{\mathrm{a}}$ & $2.04^{\mathrm{a}}$ & $16.8^{\mathrm{a}}$ \\
\hline Distilled water & yes & 5.8 & 285 & 296 & 9,170 & $31^{\circ} \leqslant \varphi \leqslant 178^{\circ}$ & $3.60^{\mathrm{a}}$ & $18.8^{\mathrm{a}}$ & $1.88^{\mathrm{a}}$ & $18.9^{\mathrm{a}}$ \\
\hline Distilled water & yes & 5.8 & 346 & 351 & 10,169 & $24^{\circ} \leqslant \varphi \leqslant 179^{\circ}$ & $3.26^{\mathrm{a}}$ & $20.1^{\mathrm{a}}$ & $1.74^{\mathrm{a}}$ & $19.4^{\mathrm{a}}$ \\
\hline Distilled water & yes & 5.8 & 389 & 391 & 10,796 & $24^{\circ} \leqslant \varphi \leqslant 163^{\circ}$ & $3.76^{\mathrm{a}}$ & $21.5^{\mathrm{a}}$ & $2.24^{\mathrm{a}}$ & $22.2^{\mathrm{a}}$ \\
\hline Distilled water & steady & 5.8 & 296 & 302 & 9,397 & & 4.09 & 20.4 & 2.61 & 18.8 \\
\hline Distilled water & steady & 5.8 & 309 & 218 & 10,520 & & 3.13 & 11.5 & 2.34 & 12.9 \\
\hline Distilled water & steady & 5.8 & 353 & 388 & 10,063 & & 4.46 & 22.5 & 2.86 & 19.4 \\
\hline Distilled water & steady & 5.8 & 381 & 406 & 10,539 & & 4.59 & 25.8 & 2.61 & 20.2 \\
\hline Distilled water & steady & 5.8 & 382 & 269 & 11,703 & & 3.45 & 14.4 & 2.49 & 14.0 \\
\hline Glycerine-water & no & 6.4 & 185 & 203 & 7,276 & $14^{\circ} \leqslant \varphi \leqslant 138^{\circ}$ & $3.49^{\mathrm{a}}$ & $12.4^{\mathrm{a}}$ & $2.06^{\mathrm{a}}$ & $15.2^{\mathrm{a}}$ \\
\hline Glycerine-water & no & 6.4 & 232 & 252 & 8,191 & $7^{\circ} \leqslant \varphi \leqslant 126^{\circ}$ & $3.82^{\mathrm{a}}$ & $14.6^{\mathrm{a}}$ & $2.28^{\mathrm{a}}$ & $14.6^{\mathrm{a}}$ \\
\hline Glycerine-water & no & 6.4 & 284 & 316 & 8,980 & $-1.5^{\circ} \leqslant \varphi \leqslant 122^{\circ}$ & $4.00^{\mathrm{a}}$ & $19.1^{\mathrm{a}}$ & $2.31^{\mathrm{a}}$ & $17.6^{\mathrm{a}}$ \\
\hline Glycerine-water & yes & 6.4 & 282 & 310 & 9,004 & $15^{\circ} \leqslant \varphi \leqslant 166^{\circ}$ & $3.79^{\mathrm{a}}$ & $19.3^{\mathrm{a}}$ & $2.00^{\mathrm{a}}$ & $19.3^{\mathrm{a}}$ \\
\hline Glycerine-water & yes & 6.4 & 337 & 364 & 9,873 & $36^{\circ} \leqslant \varphi \leqslant 153^{\circ}$ & $4.09^{\mathrm{a}}$ & $20.4^{\mathrm{a}}$ & $2.44^{\mathrm{a}}$ & $19.8^{\mathrm{a}}$ \\
\hline Glycerine-water & yes & 6.4 & 361 & 407 & 10,112 & $12^{\circ} \leqslant \varphi \leqslant 166^{\circ}$ & $4.06^{\mathrm{a}}$ & $22.3^{\mathrm{a}}$ & $2.32^{\mathrm{a}}$ & $20.8^{\mathrm{a}}$ \\
\hline Glycerine-water & steady & 6.4 & 189 & 201 & 7,418 & & 3.43 & 11.3 & 2.11 & 14.9 \\
\hline Glycerine-water & steady & 6.4 & 237 & 251 & 8,322 & & 3.67 & 13.6 & 2.27 & 14.5 \\
\hline Glycerine-water & steady & 6.4 & 275 & 306 & 8,846 & & 3.77 & 18.4 & 2.25 & 15.4 \\
\hline Glycerine-water & steady & 6.4 & 339 & 367 & 9,883 & & 4.29 & 21.9 & 2.50 & 18.5 \\
\hline Glycerine-water & steady & 6.4 & 362 & 416 & 10,072 & & 4.59 & 25.0 & 2.79 & 21.8 \\
\hline
\end{tabular}

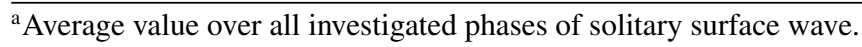



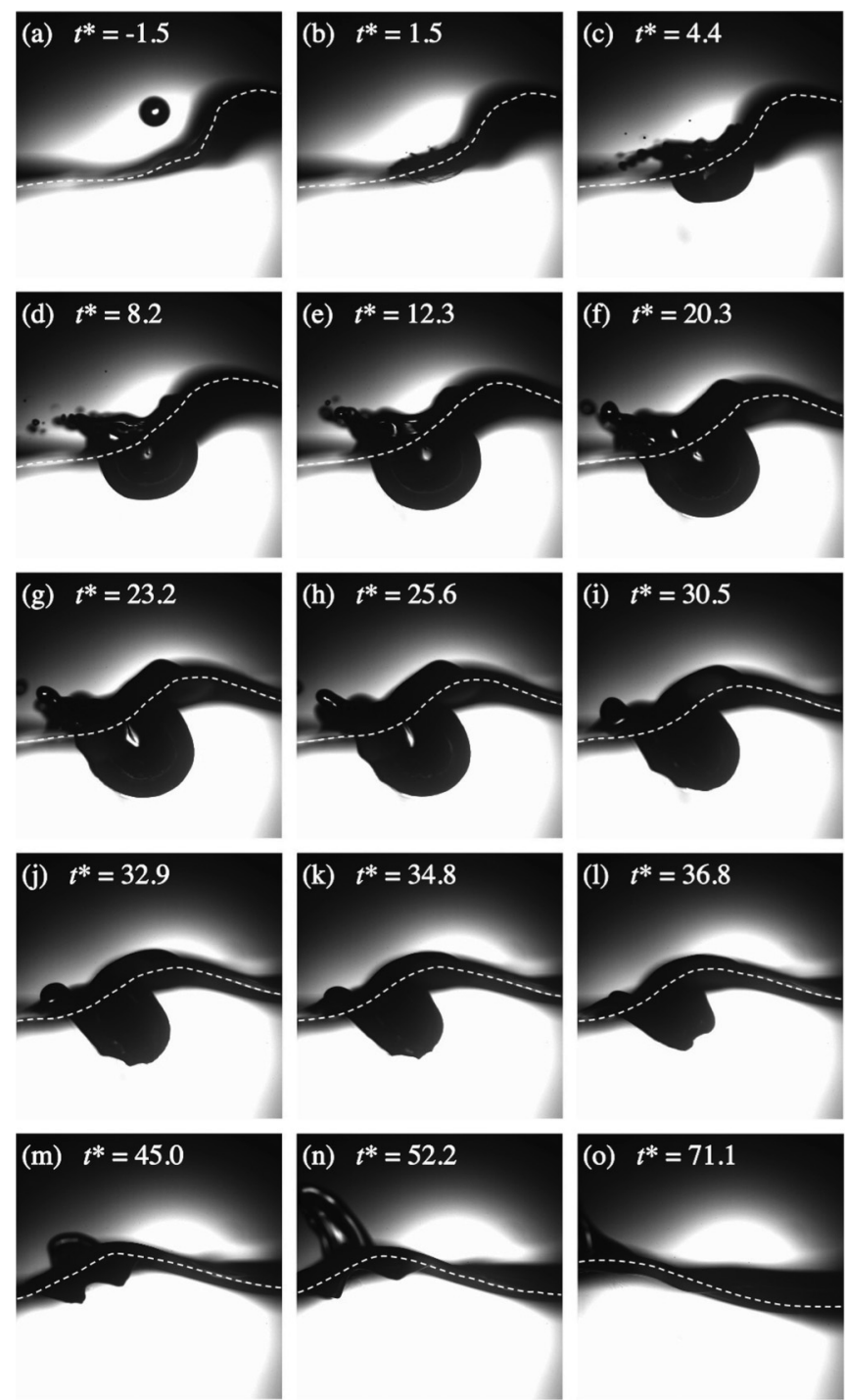

FIG. 2. Experimental results of the cavity dynamics at different nondimensional time instants after impingement onto a solitary surface wave for distilled water $(\mathrm{We}=331, \mathrm{Fr}=349, \mathrm{Re}=9845$, $\overline{h^{*}}=5.8, \varphi=21^{\circ}, U_{w}^{*}=0.15$, and $\left.A_{w}^{*}=1.94\right)$. The dashed line represents the surface of the liquid wall film.

liquid surface films the combined influence of the inclined film surface (i.e. amplitude and phase of the wave) and of the velocity distribution of the surface film - introducing a relative velocity component upon impingement and a continuous motion of the film during the cavity evolution-will become clear. A selection of the many performed impingement experiments are presented in more detail hereafter. The impingement parameters of these experiments are given in Table II.

\section{A. Experimental outcomes: Influence of a solitary surface wave on the cavity dynamics}

Figure 2 presents the experimentally obtained evolution of the cavity that is formed after impingement of a distilled water drop onto a solitary surface wave, the latter generated on top of an undisturbed film with thickness $\overline{h^{*}}=5.8$. The surface wave is defined by a nondimensional propagation velocity of
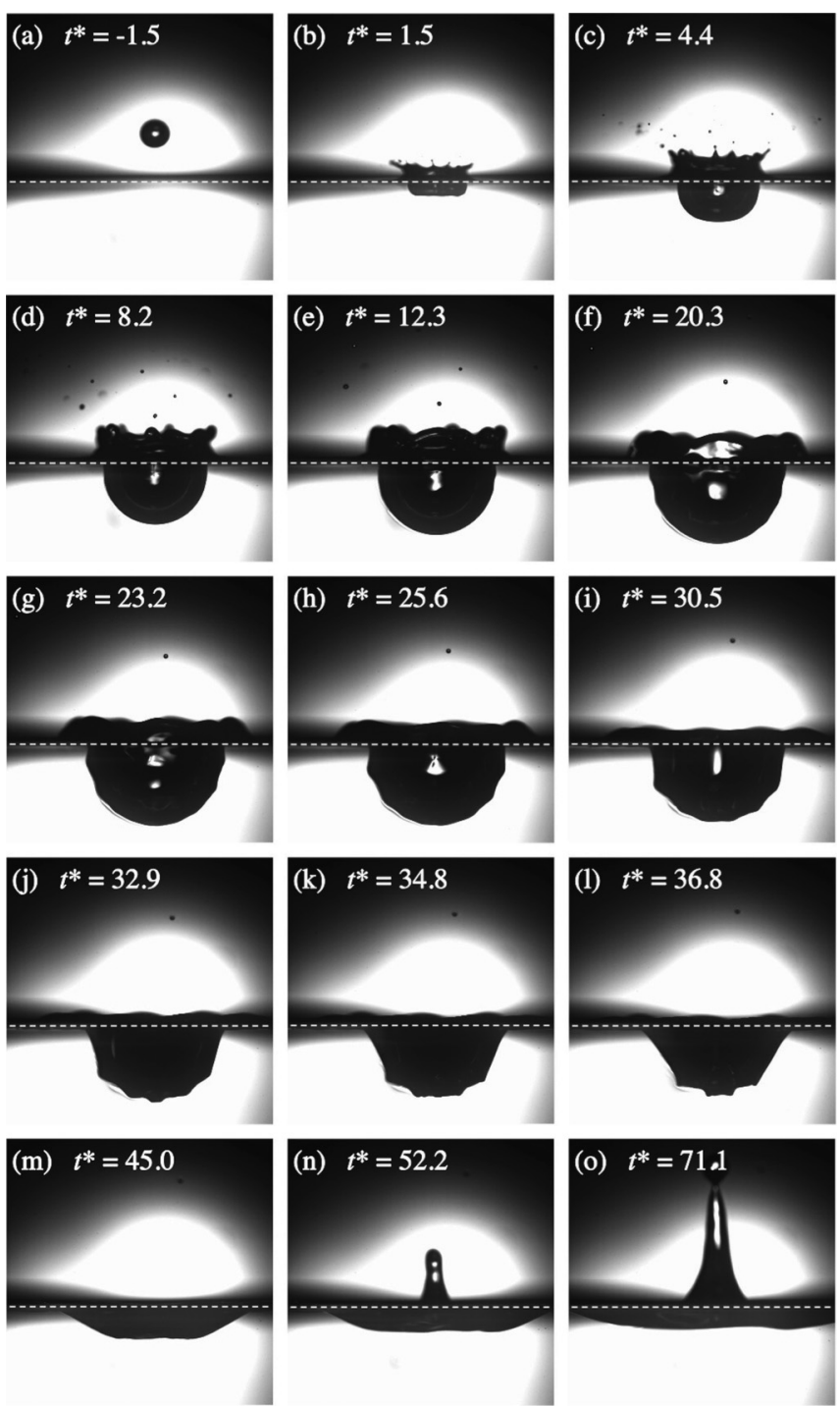

FIG. 3. Experimental results of the cavity dynamics at different nondimensional time instants after impingement onto a steady liquid film for distilled water $\left(\mathrm{We}=353, \mathrm{Fr}=338, \mathrm{Re}=10063\right.$, and $\overline{h^{*}}=$ 5.8). The dashed line represents the surface of the liquid wall film.

$U_{w}^{*}=U_{w} / U_{a b s}=0.15$ and a nondimensional amplitude of $A_{w}^{*}=A_{w} / D=1.94$. The drop impinges on a wave phase of $\varphi=21^{\circ}$, hence at the leading edge of the sinusoidal surface wave. The horizontal wave propagation velocity increases the absolute velocity of the drop at impingement by about $1 \%$. It has to be noted that the wave propagation direction is from right to left in the images presented hereafter. For a clear comparison the time evolution of the cavity for a distilled water drop onto a steady liquid surface film with equal thickness is presented in Fig. 3 for the same time instants. A direct comparison of both figures leads to a clear identification of the influences of the surface wave propagation and of the velocity distribution inside the surface film on the cavity dynamics.

A first observation is the distinct (a)symmetry of the cavity: for the steady liquid film the cavity develops in a highly symmetric way, whereas the solitary surface wave drastically changes the cavity dynamics. For both impingements the prompt splash mechanism leads to an almost immediate 
formation of very small jets and secondary droplets upon impingement. Keeping in mind that in Fig. 2 the solitary wave is moving to the left, two principles can be made responsible for the more vigorous prompt splash mechanism in the wave direction. First of all the absolute impingement velocity vector of the drop is inclined in the downstream direction, hence more kinetic energy is distributed into this direction, directly leading to a stronger downstream-directed prompt splash. Second, the drop impinges onto the leading edge of the wave, due to which the upcoming wave front prohibits a prompt splash appearance at the upstream side of the increasing corona. This upcoming wave front not only changes the prompt splash mechanism, it also influences the growth of the corona at the upstream side [see Figs. 2(c) and 2(d)].

The formation of the cavity is also directly influenced by the solitary wave propagation. The top of the radially increasing and penetrating cavity propagates in the downstream direction with approximately the solitary wave velocity $U_{w}^{*}$. In combination with a decreasing horizontal velocity of the film liquid for increasing depth this leads to an additional inclination of the radially expanding cavity in the wave propagation direction, thereby increasing its inclination angle at subsequent time instants, Figs. 2(d)-2(h).

Owing to the influence of gravity that acts on the expanding rim, the rim falls back onto and merges with the solitary surface wave, leading to the formation of the capillary waves at the inner side of the cavity. Because the rim can expand freely at the downstream side of the cavity, the interaction of the impinging rim with the surface wave is strong, thereby initiating a clear capillary wave train at this side of the cavity. At the upstream side of the cavity, however, no capillary wave train appears in Fig. 2(h). This is the direct result of the interaction of the rim with the upcoming wave front, thereby prohibiting a distinct rim formation. These observations are not observed for the impingement onto the steady liquid film. For the latter case a clear capillary wave train is present at both sides of the cavity at this stage of the impingement process [Fig. 3(g)]. The downward motion of the capillary wave train at the downstream side of the cavity in Fig. 2(h), in combination with the surface tension forces acting on the cavity, changes the shape of the cavity at this side from hemispherical to conical. The absence of the capillary waves at the upstream side of the cavity results in a minor change of the hemispherical shape of the cavity. The subsequent asymmetric receding of the cavity clearly increases its inclination, Fig. 2(1). The high surface tension forces and pressure differences built up at the bottom of the cavity during its receding phase are no longer centered at one point. This leads to an inclined cavity retraction and a curved and inclined Worthington jet. Note that due to the motion of the complete cavity in the wave propagation direction during its expansion and receding phase, the Worthington jet is formed downstream from the point of drop impingement.

\section{B. Behavior of typical time and length scales}

\section{Repeatability}

In the preceding paragraphs we focused mainly on the qualitative comparison of the experimental results for singledrop impingements onto steady liquid films and solitary
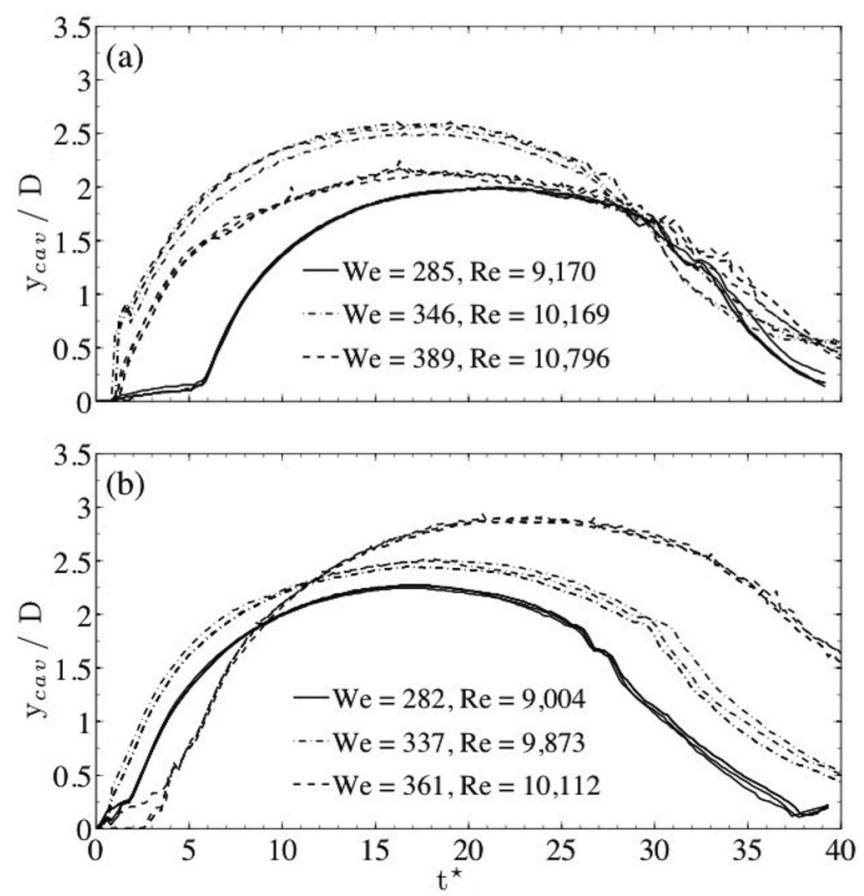

FIG. 4. Repeatability study of the cavity depth evolution in time upon impingement onto a solitary surface wave. (a) Distilled water, $\varphi=20^{\circ}$; (b) glycerine-water, $\varphi=26^{\circ}$. The various impingement parameters for both liquids are listed in Table III.

surface waves. For an even better understanding of the effect of a wavy surface on the cavity dynamics, the time evolution of the two most important cavity length scales, i.e. the cavity depth and diameter, were investigated in detail as well. The cavity depth is measured at its lowest point observed in the shadowgraph recordings, whereas the diameter of the cavity is determined at a vertical position of $50 \%$ of the maximum cavity depth, hence $y_{\text {cav }}^{*} / y_{\text {cav, } \max }^{*}=0.5$. To assure both qualitative and quantitative trustful outcomes of the impingements onto the solitary surface waves, each measurement configuration is repeated five times, after which its deviation from the mean is calculated. The time evolutions of the depths and diameters of the cavities for the repeated configurations are shown in Figs. 4 and 5 for both investigated liquids. In each figure three curves per Weber number are presented for the impingement upon the large amplified surface wave, hence $U_{w}^{*}=0.15$ and $A_{w}^{*}=1.94$ for distilled water and $U_{w}^{*}=0.13$ and $A_{w}^{*}=2.14$ for the glycerine-water mixture. The impingement parameters are defined in Table III. The figures not only show that there exists a very high repeatability of the cavity outcomes for single-drop impingements upon the investigated solitary waves, they also present that the amplification of the waves, hence their velocity and amplitude, are approximately equal for each measurement. The mean spreading in both length scales is also presented in Table III and lies for most cases below $5 \%$.

\section{Influence of the phase and amplification of the surface wave upon impingement}

In this section the influences of the phase of the wave, hence its inclination, and of the relative surface film motion 

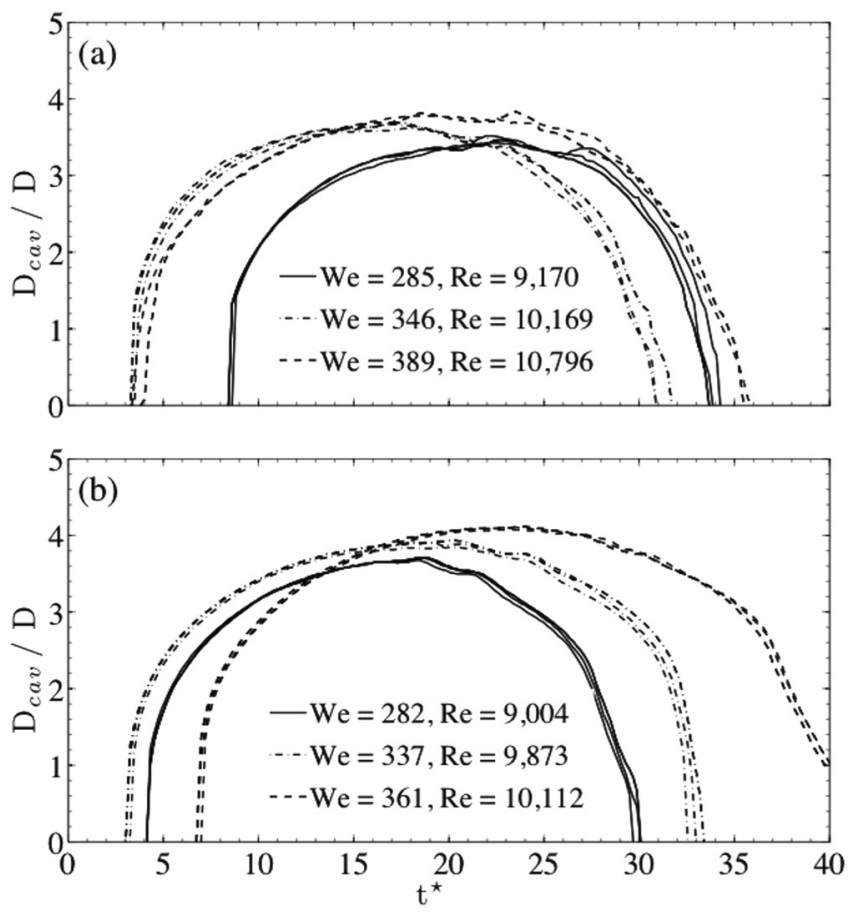

FIG. 5. Repeatability study of the cavity diameter evolution in time upon impingement onto a solitary surface wave. (a) Distilled water, $\varphi=20^{\circ}$; (b) glycerine-water, $\varphi=26^{\circ}$. The various impingement parameters are listed in Table III.

on the time evolution of the cavity are presented. Plots of the typical length scales of the cavity associated with spray impingement (the nondimensional depth $y_{\text {cav }}^{*}$ and the nondimensional diameter $D_{\text {cav }}^{*}$ ) are shown for two investigated surface wave amplifications in Figs. 6 and 7 and in Figs. 8 and 9 respectively. As a reference, the time evolutions of the same length scales for the impingements upon the steady surface films of equal thickness are added as well.

a. Effect of wave phase at impingement on cavity dynamics. The influence of the phase of the wave on the time evolution of the cavity depth is relatively small for the nonamplified surface wave (Fig. 6), as the curves for the different phases lie close to the curve of the impingement onto the steady surface film. The reason behind this observation is the low velocity $\left(U_{w}^{*}=0.07\right)$ and amplitude $\left(A_{w}^{*}=0.69\right)$ of the solitary wave, resulting in a relatively small inclination of the surface film upon impingement. The solitary wave has therefore no direct influence on the dynamics of the cavity depth. The same conclusion can be drawn from the total diameter evolution of the cavity as presented in Figs. 7(a) and 7(b), although here a slight divergence from the reference curve (i.e., the solid line) is observed for increasing phases.

An interesting and distinct influence of the phase of the solitary surface wave is found for the relative diameters of the cavity, Figs. 7(c) and 7(d). The left-directed propagation of the solitary surface wave induces a surface film velocity in the same direction. During the expansion of the cavity this film velocity opposes a strong increase of the radial diameter at the upstream side of the cavity, whereas it enhances the diameter increase at its downstream side as a result of the equivalent propagation direction of the cavity expansion and the solitary wave. Even for a solitary wave with a low amplification, hence with a low propagation velocity, this leads to smaller values of the expansion rates and maximum relative diameters at the upstream side of the cavity than is found for the impingement upon the steady surface film. At the same instant, the values of the maximum diameters at the downstream side are up to about $30 \%$ higher in comparison to the steady surface film impingement. The opposing velocities at the upstream side of the cavity also lead to a faster conversion of the kinetic energy into surface tension energy and dissipated energy, thereby initiating a somewhat earlier receding of the cavity at this side. This again results in a larger difference between the values of the relative diameters at both sides of the cavity during the receding phase. It is observed that these differences decrease for larger phases of the wave at impingement. This phenomenon is linked to the position of the solitary wave upon drop impingement. For higher wave phases the drop impinges on the top or on the receding part of the wave where the influence of and interaction with the solitary wave is weaker. The curves for both relative diameters therefore approach again the curve of the cavity diameter for the steady liquid film case. This is for example observed for $\varphi=173^{\circ}$, for which the differences in the depth [Fig. 6(b)], the total [Fig. 7(b)], and the relative [Fig. $7(\mathrm{~d})]$ diameter of the cavity are negligible when compared with the outcomes for the impingement onto the steady liquid film.

b. Effect of wave amplification on cavity dynamics. A stronger amplification of the solitary surface wave leads to distinct changes of the curves of the depth and the diameter evolution for different phases of the wave. In Fig. 8 it is observed that for all investigated phases in the range between $0^{\circ} \leqslant \varphi \leqslant 180^{\circ}$ a clear and strong decrease of the slope of the curves $y_{\text {cav }}^{*}$ vs time during the penetration phase of the cavity is found. This means that for a higher wave amplification the

TABLE III. Mean spreading of the depths and absolute diameters of the cavities after five repeated measurements.

\begin{tabular}{|c|c|c|c|c|c|c|c|}
\hline \multirow[b]{2}{*}{ Liquid } & \multirow[b]{2}{*}{ Amplification } & \multirow[b]{2}{*}{$\varphi(\operatorname{deg})$} & \multirow[b]{2}{*}{ We } & \multirow[b]{2}{*}{$\mathrm{Fr}$} & \multirow[b]{2}{*}{$\operatorname{Re}$} & \multicolumn{2}{|c|}{ Standard deviation in } \\
\hline & & & & & & depth $(\%)$ & diameter $(\%)$ \\
\hline Distilled water & large & 20 & 285 & 296 & 9170 & 2.8 & 3.3 \\
\hline Distilled water & large & 20 & 346 & 351 & 10169 & 4.0 & 4.3 \\
\hline Distilled water & large & 20 & 389 & 391 & 10796 & 4.7 & 2.0 \\
\hline Glycerine-water & large & 26 & 282 & 310 & 9004 & 2.5 & 4.5 \\
\hline Glycerine-water & large & 26 & 337 & 364 & 9873 & 6.1 & 5.4 \\
\hline Glycerine-water & large & 26 & 361 & 407 & 10112 & 3.7 & 4.5 \\
\hline
\end{tabular}



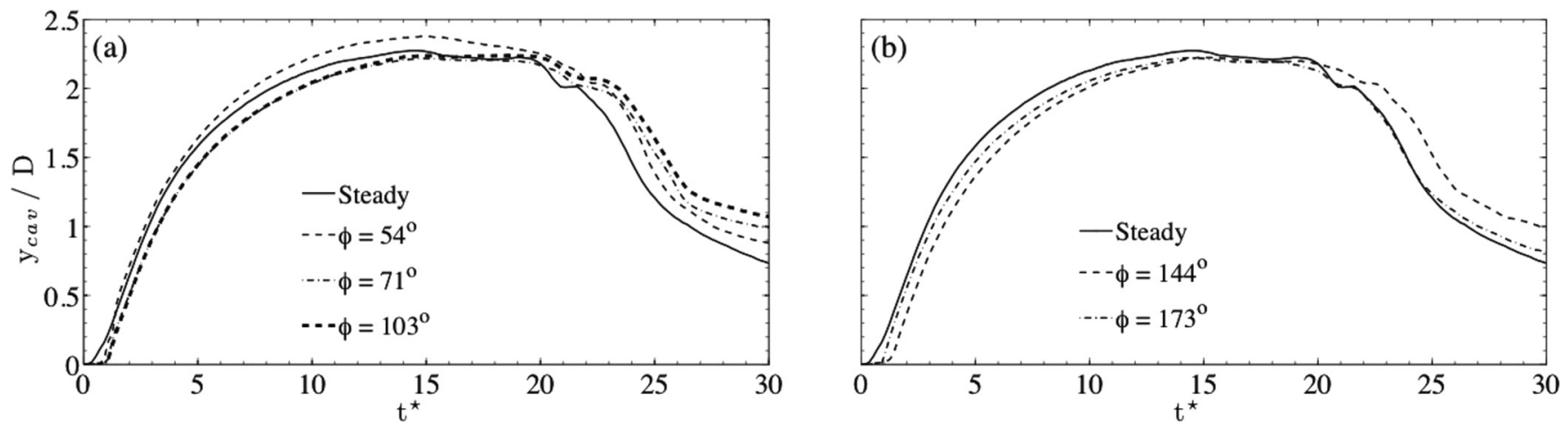

FIG. 6. Time evolution of the depth of the cavity as a function of the phase of the solitary surface wave upon impingement onto the nonamplified surface wave ( $\mathrm{We}=232, \operatorname{Re}=8191, \overline{h^{*}}=6.4, U_{w}^{*}=0.07$, and $A_{w}^{*}=0.69$ ). (a) $\varphi=54^{\circ}, 71^{\circ}$, and $103^{\circ} ;$ (b) $\varphi=144^{\circ}$ and $173^{\circ}$. Solid line: reference case with steady surface film of $\overline{h^{*}}=6.4$. The various impingement parameters for glycerine-water are listed in Table II.

vertical penetration velocity of the cavity into the surface film is smaller, thereby leading to lower values of the maximum depth of the cavity that are in addition also reached at later time instants than was found for the nonamplified surface wave. These phenomena result from a stronger interaction between the expanding cavity and the upcoming wave front for this range of wave phases. The vector of the total kinetic energy upon impingement has a more horizontal inclination. Due to the lower energy available in the downward direction the cavity cannot penetrate into the surface film as deep as for the impingement upon a steady surface film. Together with the relatively large solitary wave velocity of $U_{w}^{*}=0.13$ this leads to a stronger reduction of the distinct radial expansion of the cavity at its upstream side and higher diameter values at the downstream side of the cavity than was observed for approximately the same phases upon impingement onto the nonamplified surface waves. This aspect is clearly visible in Fig. 9 for both the total and the relative diameters. The higher wave film velocity leads to later time instants at which the maximum values of the absolute and relative diameters are reached, as listed in Table II. Similar to the results for $\varphi=173^{\circ}$ for the nonamplified wave, the curves for the absolute and relative diameters for $\varphi=168^{\circ}$ approach the curves of the cavity diameter upon impingement onto the steady liquid film, Fig. 9(f). This is, however, not the case for the depth of the cavity for which a relatively constant distinct deviation to the steady film results is present for all investigated wave phases. Hence, in depth direction the strong interaction between the
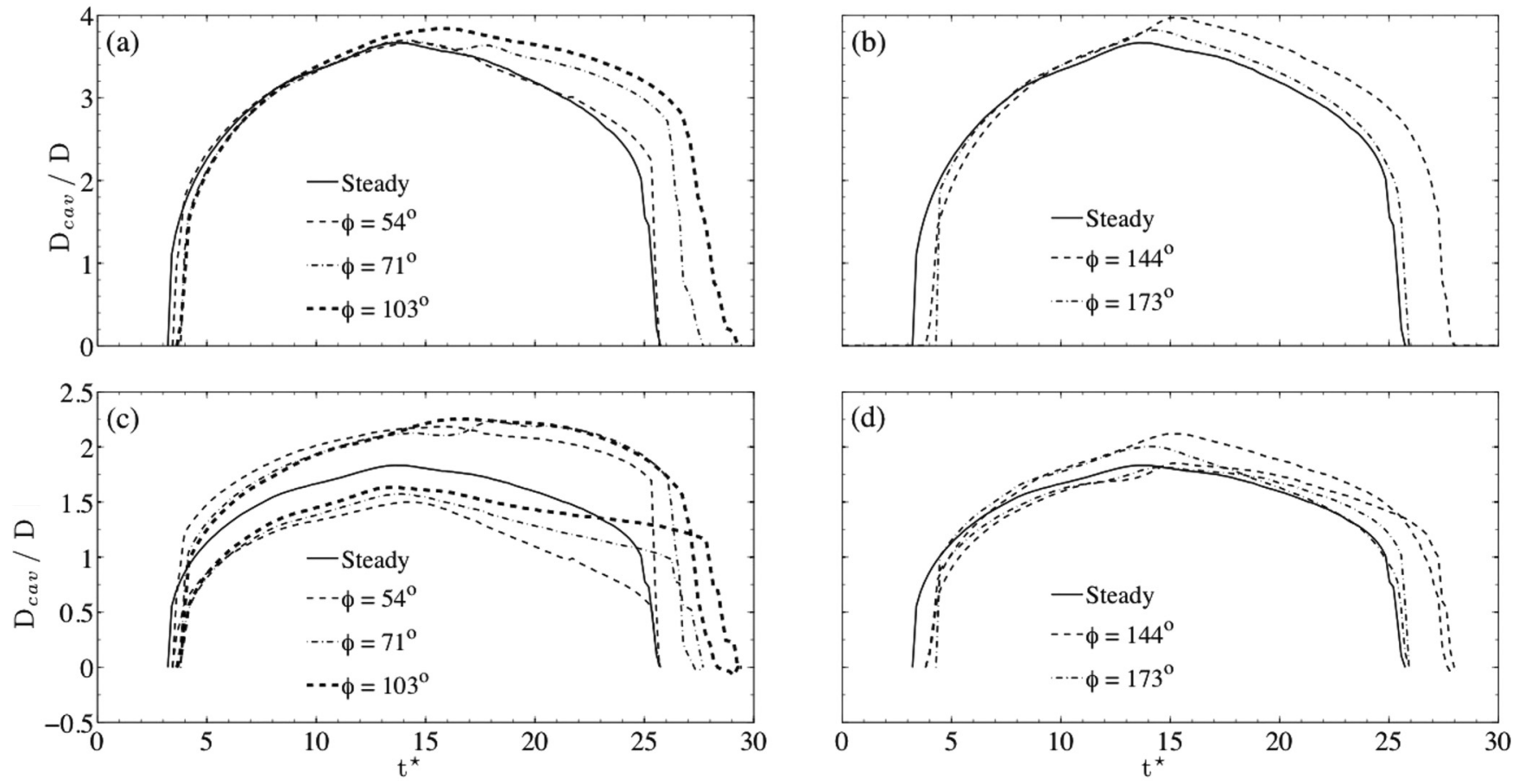

FIG. 7. Time evolution of the total (a),(b) and relative (c),(d) diameter of the cavity as a function of the phase of the solitary surface wave upon impingement onto the nonamplified surface wave (We $=232, \operatorname{Re}=8191, \overline{h^{*}}=6.4, U_{w}^{*}=0.07$, and $A_{w}^{*}=0.69$ ). (a), (c) $\varphi=54^{\circ}$, $71^{\circ}$, and $103^{\circ} ;(\mathrm{b})(\mathrm{d}) \varphi=144^{\circ}$ and $173^{\circ}$. Solid line: reference case with steady surface film of $h^{*}=6.4$. The impingement parameters for glycerine-water are listed in Table II. 

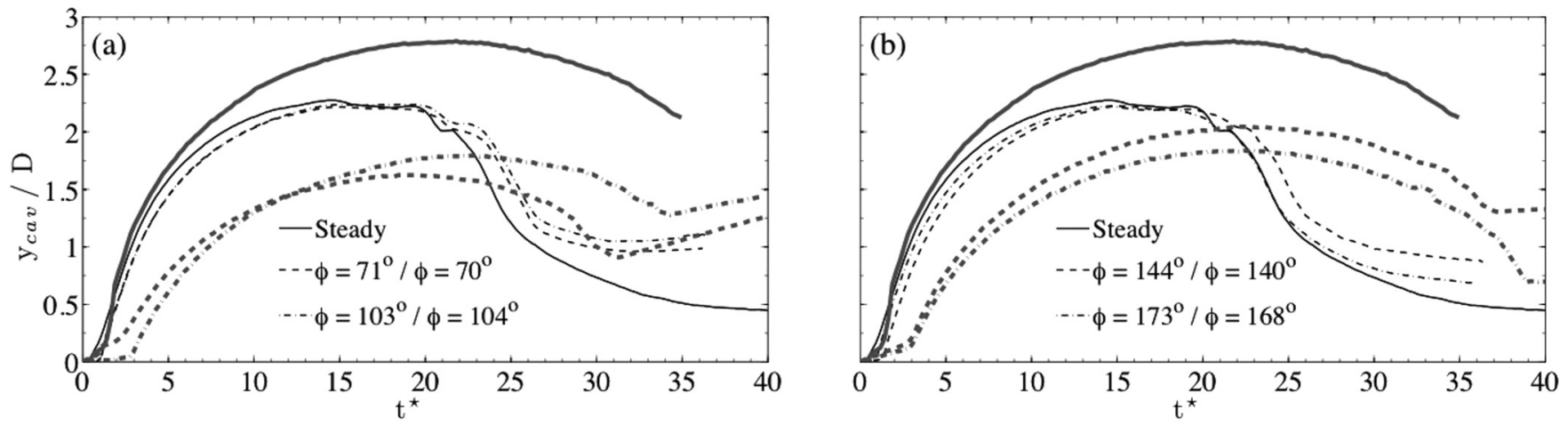

FIG. 8. Time evolution of the depth of the cavity as a function of the wave amplification for various phases of the solitary surface wave upon impingement. Thin lines: nonamplified surface wave $\left(\mathrm{We}=232, \operatorname{Re}=8191\right.$, and $\overline{h^{*}}=6.4, U_{w}^{*}=0.07, A_{w}^{*}=0.69$, and $\varphi=71^{\circ}, 103^{\circ}, 144^{\circ}$, and $173^{\circ}$ ); thick lines: amplified surface wave ( $\mathrm{We}=361, \mathrm{Re}=10112$, and $\overline{h^{*}}=6.4, U_{w}^{*}=0.13, A_{w}^{*}=2.14$, and $\varphi=70^{\circ}, 104^{\circ}, 140^{\circ}$, and $168^{\circ}$ ). Solid line: reference case with steady surface film of $\overline{h^{*}}=6.4$. The impingement parameters for glycerine-water are listed in Table II.
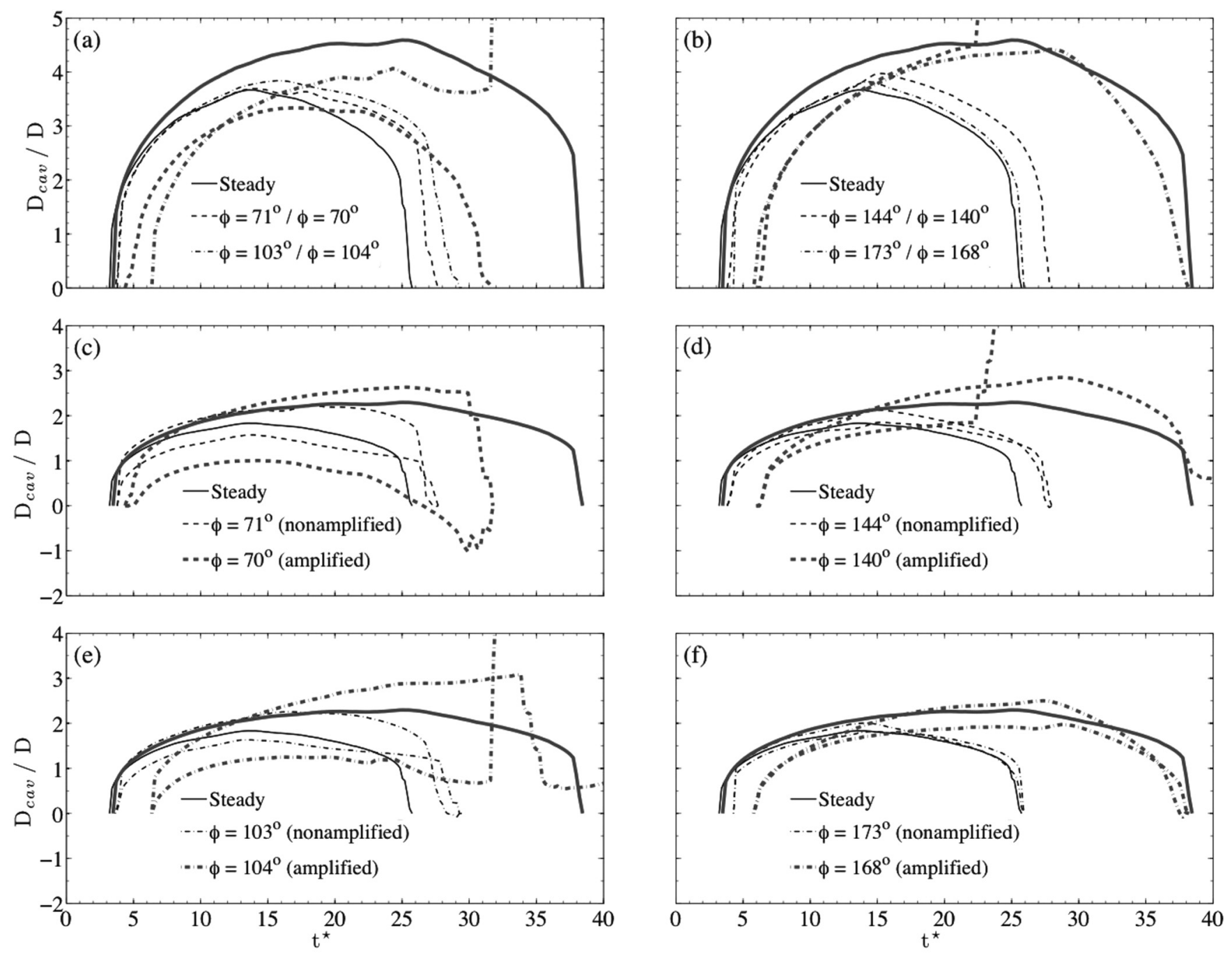

FIG. 9. Time evolution of the total (a),(b) and relative (c)-(f) diameter of the cavity as a function of the wave amplification for various phases of the solitary surface wave upon impingement. Thin lines: nonamplified surface wave $\left(\mathrm{We}=232, \operatorname{Re}=8191\right.$, and $\overline{h^{*}}=6.4, U_{w}^{*}=0.07$, $A_{w}^{*}=0.69$, and $\varphi=71^{\circ}, 103^{\circ}, 144^{\circ}$, and $\left.173^{\circ}\right)$; thick lines: amplified surface wave $\left(\mathrm{We}=361, \operatorname{Re}=10112\right.$, and $\overline{h^{*}}=6.4, U_{w}^{*}=0.13$, $A_{w}^{*}=2.14$, and $\varphi=70^{\circ}, 104^{\circ}, 140^{\circ}$, and $168^{\circ}$ ). Solid line: reference case with steady surface film of $\overline{h^{*}}=6.4$. The impingement parameters for glycerine-water are listed in Table II. 

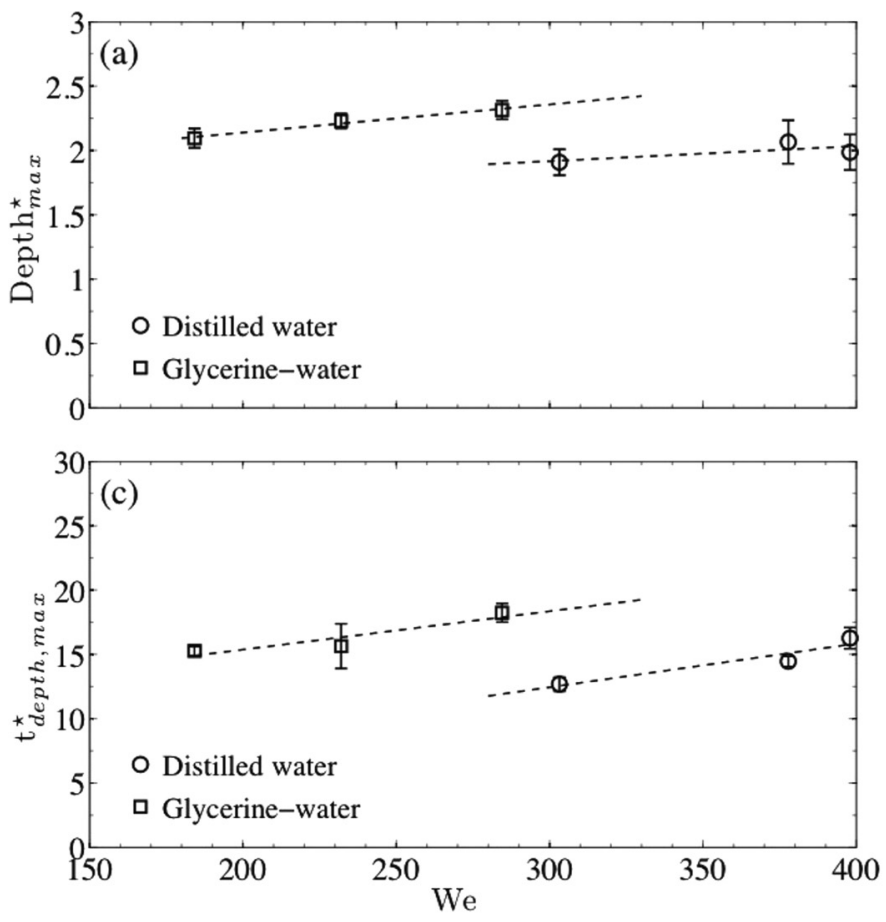
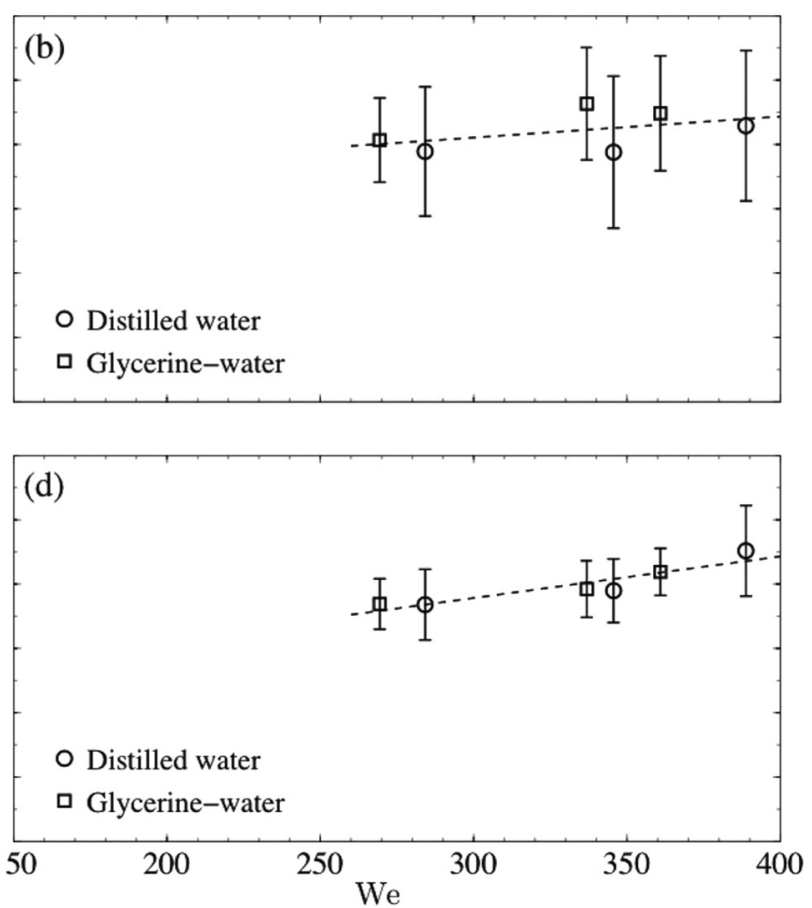

FIG. 10. Mean and $95 \%$ confidence values of the maximum depth of the cavity (a),(b) and of the time instant of maximum cavity depth (c),(d) as a function of the mean Weber number for both liquids upon impingement onto both studied solitary wave topologies. (a),(c) Nonamplified surface wave; (b),(d) amplified surface wave. The values are averaged over all investigated phases of the solitary surface wave upon impact, as listed in Table II.

cavity and the upcoming wave front is practically independent of the phase of the surface wave at the moment of impingement.

\section{Impact of a change in drop impingement velocity on the time and length scales}

In the previous section the influences of the characteristics of the liquid film on the cavity dynamics upon drop impingement were presented. They are, though, not the only parameters that should be taken into account when investigating a singledrop impingement process. The properties of the impinging drops also play a dominant role. In this section the focus lies on the drop impingement velocity and its influence on the dynamics of the formed cavity. For that reason the terminal drop velocity was varied, while the propagation velocity of the solitary surface wave remained constant. The obtained typical time and length scales are listed in Table II and presented graphically in Figs. 10 and 11 for respectively the depth and diameter of the cavity. In both figures the mean and $95 \%$ confidence values of the maximum length scales and of the time instants at which these maximum values are reached are shown as a function of the absolute Weber number. Note once more that in this paper the Weber number is calculated with the total impingement velocity (see Sec. II B).

The length and time scales of the two parameters show a clear increase with the Weber number. For higher terminal drop velocities, a larger amount of kinetic energy is available upon impingement, due to which the surface tension forces- that act on the cavity and oppose its radial expansion-can be overcome for a longer time period. This leads directly to larger expansions of the cavity and thus higher values of the maximum depth and diameter and of the associated time scales. The values all show a linear relation with the absolute Weber number for both solitary wave topologies. This specific behavior can be linked to the expansion of the cavity and the associated appearance of the surface tension forces on the cavity surface. For higher Weber numbers the radial expansion of the cavity is larger. A larger surface area of the cavity, however, results automatically in larger values of the surface tension forces acting on its free surface, thereby opposing and decelerating the further radial expansion of the cavity. This means that at each subsequent time instant upon impingement the increment of the radial cavity penetration into the surface film becomes smaller. The cavity therefore reaches a higher maximum depth and diameter. Due to the more available kinetic energy this process takes longer, hence the times at which these maximum values are reached are larger.

An interesting observation is the apparent dependency of the aforementioned parameters on the properties of the liquid for the case of the impingement upon the nonamplified solitary wave. This phenomenon is analyzed in more detail in the following section.

\section{Effect of the liquid properties on the cavity dynamics}

In [25] it was proven that the influence of the viscosity on the cavity dynamics after impingement upon a steady liquid film of finite thickness is negligible in case the Reynolds number of the impinging drop is high enough $\left(\operatorname{Re} \geqslant 5 \times 10^{3}\right)$. At such high Reynolds numbers the effect of viscosity is found to be significant only at the relatively thin boundary layer that is present below the cavity around the time instants of maximum 

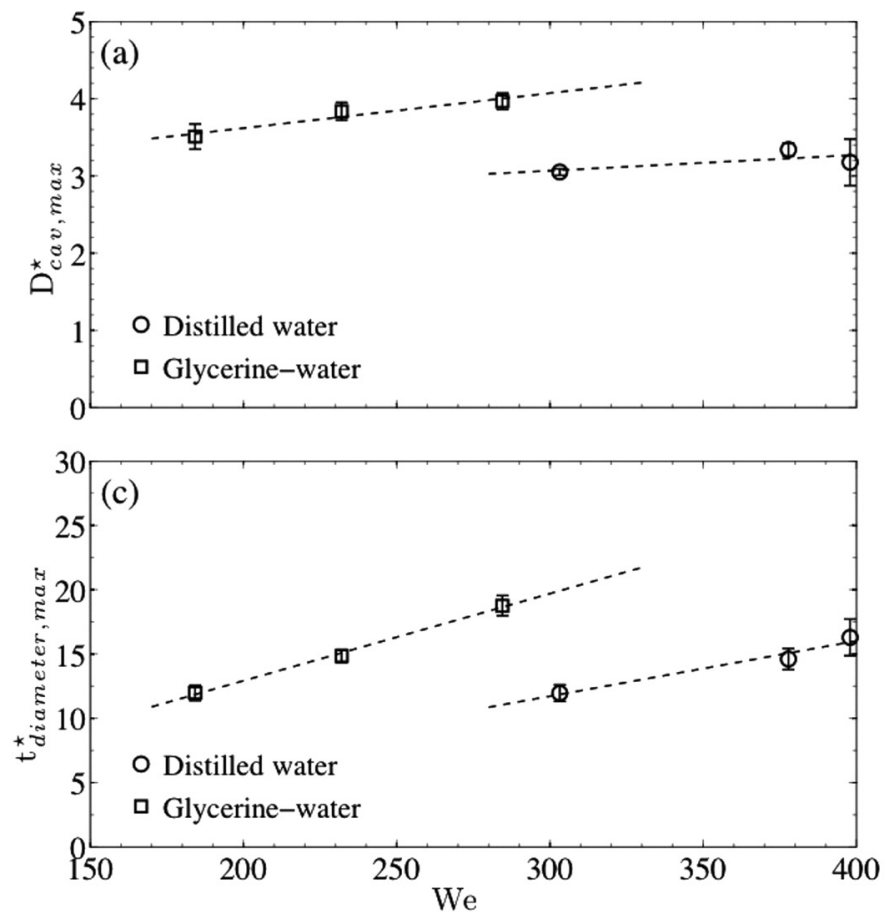
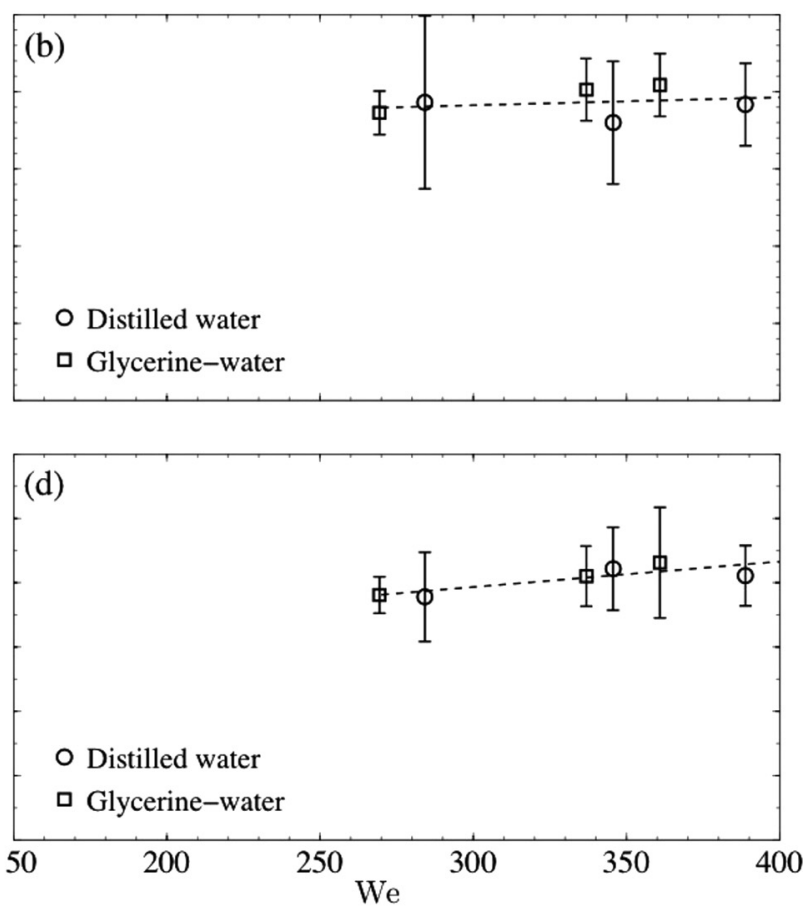

FIG. 11. Mean and 95\% confidence values of the maximum diameter of the cavity (a),(b) and of the time instant of maximum cavity diameter (c),(d) as a function of the mean Weber number for both liquids upon impingement onto both investigated solitary wave topologies. (a),(c) Nonamplified surface wave; (b),(d) amplified surface wave. The values are averaged over all investigated phases of the solitary surface wave upon impact, as listed in Table II.

cavity depth. The viscous dissipation in these layers leads to the thickening of this boundary layer, but it does not influence the outer regions and thus the cavity expansion and receding phases.

To investigate whether this statement also holds for the cavity dynamics upon an inclined and propagating surface film the impingements onto distilled water and a glycerine-water mixture of $30-70 \%$ vol were investigated, of which the properties are listed in Table I. These two liquids were chosen as their values of the surface tension are similar, whereas the ratio of the viscosities equals 0.43 . Although there exists a small difference in diameter between the drops of both liquids, it can be assumed that for the present investigation the influence of this variable is negligible. It can therefore be stated that the deviations in the outcomes for both liquids as described hereafter result solely from the difference in viscosity.

The time evolution of the total diameter, the relative diameters, and the depth of the cavity upon impingement onto various phases of the nonamplified solitary wave are shown for both liquids in respectively Figs. 12(a), 12(b)-12(d), and 13. The slopes of the curves for the depth and for the total diameter of the cavity vs time are similar for both liquids during the first stages of the expansion phase of the cavity. Yet, the maximum radial cavity expansion-both in depth and in diameter-for the glycerine-water mixture is for most investigated wave phases about $25 \%$ larger and the values are reached at later time instants. The values are plotted as a function of the absolute Weber number in Figs. 10(a) and 11(a) and in Figs. 10(c) and 11(c). Although the experiments for distilled water were performed at lower Weber numbers than was the case for the glycerine-water mixture, it can be assumed by linear extrapolation that for a constant Weber number of the impinging drop higher values of both length and time scales are obtained for the glycerine-water mixture. Furthermore, as the slopes of the curves for glycerine-water are larger, an increase of the absolute impinging Weber number has a stronger effect on the cavity dynamics.

The reason for these differences between both liquids is found in the propagation velocity and amplitude of the nonamplified solitary wave, in combination with the viscosity. The solitary surface wave has a lower propagation velocity for glycerine-water. Although this wave moves in opposite direction to the cavity expansion at the upstream cavity side, the expansion can continue at this side during a longer time period, as the wave velocity is smaller than for distilled water and thus offers less resistance to the cavity expansion per time instant. This means that the deceleration of the cavity expansion is smaller; hence it takes longer before the surface tension forces overcome the kinetic energy. The same explanation holds for the downstream side of the cavity, where for the glycerine-water mixture a stronger expansion of the cavity takes place as well, as can clearly be seen in Figs. 12(b)-12(d). These phenomena result in a wider cavity, hence a larger maximum of the depth [Fig. 10(a)] and absolute diameter of the cavity [Fig. 11(a)] and later time instants at which these maximum values are reached [Figs. 10(c) and 11(c)]. A larger value of the maximum diameter for the glycerine-water mixture, in combination with the almost identical value of the surface tension as for distilled water, therefore also leads to a later initiation of and a longer time period during which the receding of the cavity takes place and 

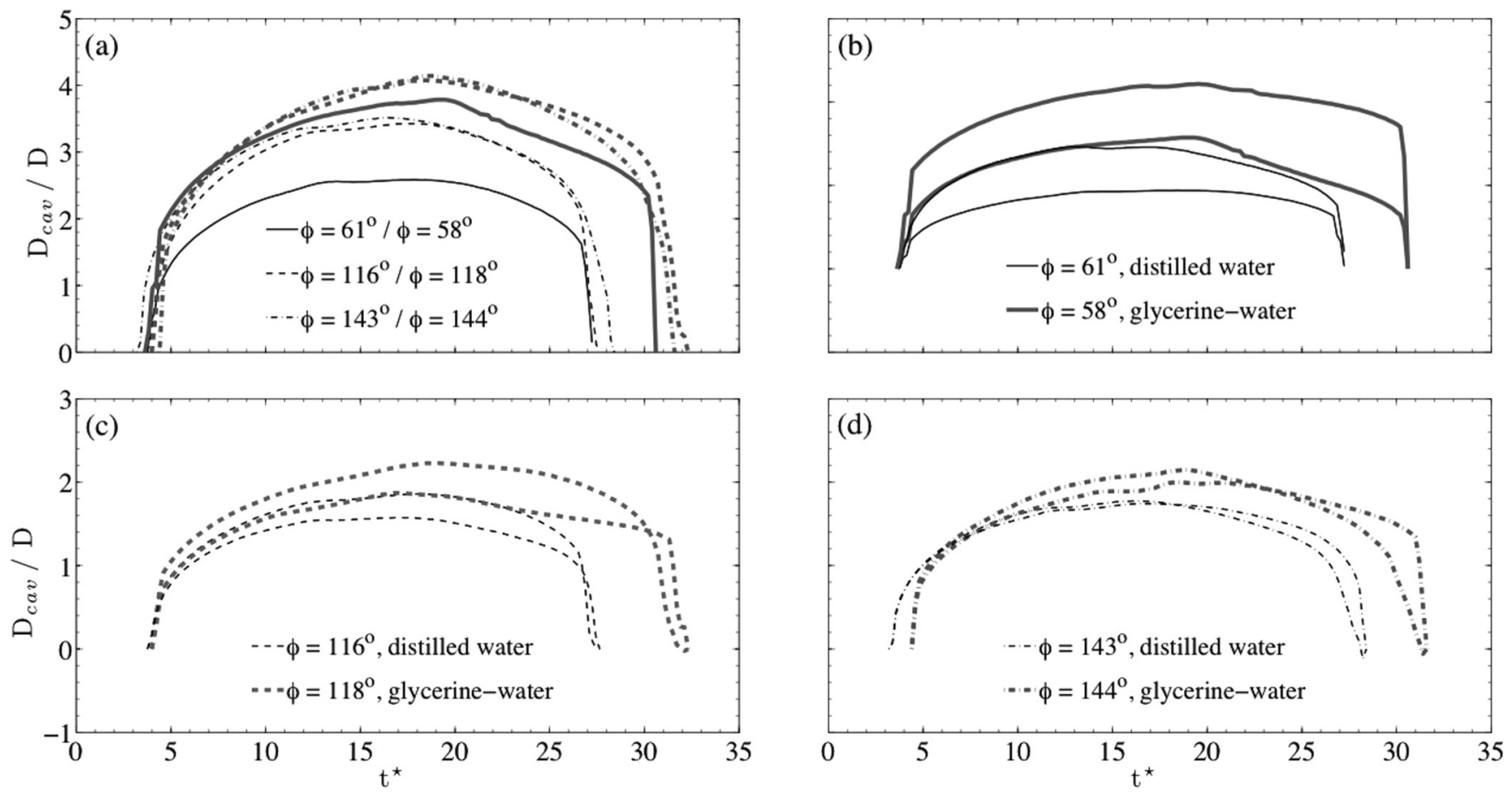

FIG. 12. Time evolution of the total (a) and relative (b)-(d) diameter of the cavity as a function of the viscosity upon impingement onto the nonamplified solitary surface wave. Thin lines: distilled water $\left(\mathrm{We}=398, \operatorname{Re}=11.458, \overline{h^{*}}=5.8, U_{w}^{*}=0.10, A_{w}^{*}=0.78\right.$, and $\varphi=61^{\circ}$, $116^{\circ}$, and $\left.143^{\circ}\right)$; thick lines: glycerine-water $\left(\mathrm{We}=284, \operatorname{Re}=8980, \overline{h^{*}}=6.4, U_{w}^{*}=0.07, A_{w}^{*}=0.69\right.$, and $\varphi=58^{\circ}, 118^{\circ}$, and $\left.144^{\circ}\right)$.

thus a later start of the retraction of the cavity. Interesting to note is that for both liquids the same behavior of the depth and of the diameter evolution is seen for increasing wave phases upon impingement. Whereas the depth evolution of the cavity remains nearly unaffected by the variation of the wave phase for both liquids (Fig. 13), a decrease of the differences between the relative diameters at both sides of the cavity is seen to occur for increasing wave phases, Figs. 12(b)-12(d).

The influence of the viscosity on the cavity dynamics, discussed above for the impingement onto the nonamplified

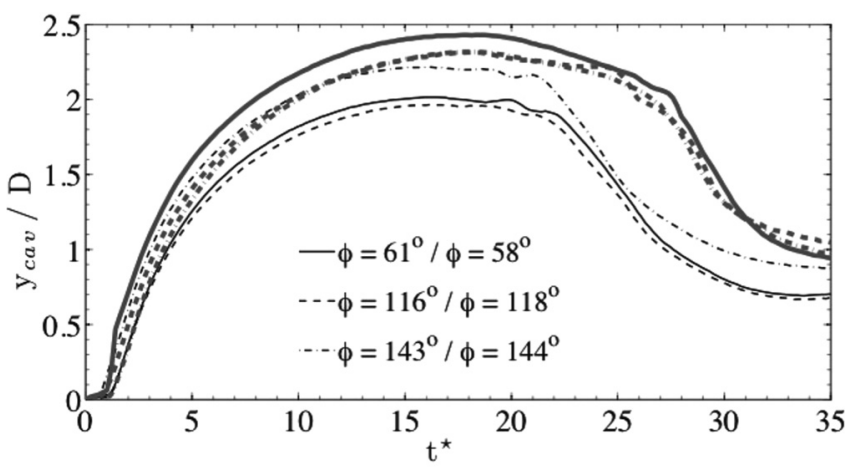

FIG. 13. Time evolution of the depth of the cavity as a function of the viscosity upon impingement onto the nonamplified solitary surface wave. Thin lines: distilled water $(\mathrm{We}=398, \mathrm{Re}=11.458$, $\overline{h^{*}}=5.8, U_{w}^{*}=0.10, A_{w}^{*}=0.78$, and $\varphi=61^{\circ}, 116^{\circ}$, and $\left.143^{\circ}\right)$; thick lines: glycerine-water $\left(\mathrm{We}=284, \mathrm{Re}=8980, \overline{h^{*}}=6.4, U_{w}^{*}=\right.$ $0.07, A_{w}^{*}=0.69$, and $\varphi=58^{\circ}, 118^{\circ}$, and $144^{\circ}$ ). solitary surface wave, is seen to disappear completely for drop impingements onto surface waves with a higher amplification. The values of the length scales [Figs. 10(b) and 11(b)] and time scales [Figs. 10(d) and 11(d)] for both liquids follow the same linear relation with the absolute Weber number. Smaller differences of the solitary wave amplification and higher values of the Reynolds number for both liquids lead to negligible effects of the viscosity on the cavity dynamics, in agreement with the results presented in [25] for the impingement onto steady surface films of finite thickness.

\section{CONCLUSIONS}

The present study was devoted to gain insight into the influences of the film surface waviness and film velocities on the dynamics of a single-drop impingement process at high Froude and Weber numbers. Experimental observations were performed, thereby focusing in particular on the evolution of the cavity that was formed upon drop impingements onto a dynamic liquid surface film. One particular kind of surface wave topology was studied and discussed in this paper: the so-called "travelling solitary surface wave." The combined effects of the surface wave velocity, surface wave amplitude, and the velocity of the liquid layer were investigated by impingement experiments onto various wave phases. To study a possible influence of the Weber number on the impingement outcomes, the drop impingement velocity and viscosity of the liquid were varied as well.

The experiments revealed a distinct effect of the dynamic surface film on the cavity dynamics, particularly for the diameter evolution in time. An asymmetrical distribution of 
the radial expansion velocity along the cavity's circumference appeared right from the initial phase of the crater expansion and remained present throughout the complete expansion and receding phase. The shadowgraph recordings showed that the top of the expanding cavity propagated in horizontal direction with approximately the solitary wave velocity. In combination with a smaller horizontal motion of the cavity with increasing film depth, this led to a strong and in time increasing inclination of the cavity in the wave propagation direction. At the azimuthal cavity locations that lay upstream of the drop impingement point a lower radial expansion velocity than for the undisturbed film was therefore identified. The opposite was observed for azimuthal cavity locations lying downstream of this point, for which a larger radial expansion velocity was measured. Depending on the local inclination of the surface wave in the vicinity of the impingement point the overall cavity diameter could deviate from the square-root law predicted in [27] — in particular for larger wave amplitudes and propagation velocities. The typical length and time scales associated with the maximum depth and maximum diameter of the cavity increased linearly with growing absolute Weber number.

\section{ACKNOWLEDGMENTS}

The authors would like to thank the Centre of Smart Interfaces (CSI) of the Technische Universität Darmstadt for sponsoring the exchange programme of $\mathrm{M}$. CharbonneauGrandmaison in 2009 by the RISE-programme (Research Internships in Science and Engineering) of the DAAD (Deutscher Akademischer Austauschdienst). We furthermore would like to thank the reviewers for their specific comments.
[1] V. Mehdi-Nejad, J. Mostaghimi, and S. Chandra, Phys. Fluids 15, 173 (2003).

[2] P. Lenard, Ann. Phys. (NY) 352, 463 (1915).

[3] Z. Levin and P. V. Hobbs, Philos. Trans. R. Soc. A 269, 555 (1971).

[4] W. D. Ellison, Agric. Eng. 25, 131 (1944).

[5] M. Rein, Fluid Dyn. Res. 12, 61 (1993).

[6] A. Prosperetti, J. Fluid Mech. 100, 333 (1980).

[7] Y. K. Cai, Exp. Fluids 7, 388 (1989).

[8] D. S. Chapman and P. R. Critchlow, J. Fluid Mech. 29, 177 (1967).

[9] F. Rodriguez and R. J. Mesler, J. Colloid Interface Sci. 121, 121 (1988).

[10] B. Peck and L. Sigurdson, Phys. Fluids 6, 564 (1994).

[11] M. Santini, S. Fest-Santini, and G. E. Cossali, Exp. Fluids 54, 1593 (2013).

[12] H. N. Oguz and A. Prosperetti, J. Fluid Mech. 228, 417 (1991).

[13] D. Morton, M. Rudman, and L. Jong-Leng, Phys. Fluids 12, 747 (2000).

[14] Z. Mohamed-Kassim and E. K. Longmire, Phys. Fluids 16, 2170 (2004).
[15] E. Berberović, N. P. van Hinsberg, S. Jakirlic, I. V. Roisman, and C. Tropea, Phys. Rev. E 79, 036306 (2009).

[16] L. J. Leng, J. Fluid Mech. 427, 73 (2001).

[17] Q. Deng, A. V. Anilkumar, and T. G. Wang, J. Fluid Mech. 578, 119 (2007).

[18] A. Bisighini, G. E. Cossali, C. Tropea, and I. V. Roisman, Phys. Rev. E 82, 036319 (2010).

[19] H. C. Pumphrey et al., J. Acoust. Soc. Am. 85, 1518 (1989).

[20] A. Prosperetti and H. N. Oguz, Annu. Rev. Fluid Mech. 25, 577 (1993).

[21] S. L. Zbhankova and A. V. Kolpakov, Fluid Dyn. 25, 470 (1990).

[22] G. Leneweit et al., J. Fluid Mech. 543, 303 (2005).

[23] T. Okawa, T. Shiraishi, and T. Mori, Exp. Fluids 44, 331 (2008).

[24] B. Ray, G. Biswas, and A. Sharma, Commun. Comput. Phys. 11, 1386 (2012).

[25] I. V. Roisman, N. P. van Hinsberg, and C. Tropea, Phys. Rev. E 77, 046305 (2008).

[26] J. Fukai et al., Phys. Fluids 7, 236 (1995).

[27] A. L. Yarin and D. A. Weiss, J. Fluid Mech. 283, 141 (1995).

[28] C. Weiss and A. L. Yarin, J. Fluid Mech. 385, 229 (1999).

[29] N. P. van Hinsberg et al., J. Colloid Interface Sci. 350, 336 (2010). 